\title{
Detection of serotonin, melatonin and their metabolites in honey
}

Tae-Kang Kim ${ }^{1,2}$, Venkatram R. Atigadda ${ }^{1}$, Pawel Brzeminski ${ }^{1,4}$, Adrian Fabisiak ${ }^{1,4}$, Edith K. Y. Tang ${ }^{3}$,

Robert C. Tuckey ${ }^{3}$, Russel J. Reiter ${ }^{5}$, Andrzej T. Slominski ${ }^{1,2 *}$

${ }^{1}$ Department of Dermatology, University of Alabama at Birmingham, ${ }^{2} \mathrm{VA}$ Medical Center, Birmingham, AL 35294, USA, ${ }^{3}$ School of Molecular Sciences, The University of Western Australia, Perth, WA 6009, Australia, ${ }^{4}$ Department of Chemistry, University of Warsaw, Pasteura 1, 02-093 Warsaw, Poland, ${ }^{5}$ Department of Cellular and Structural Biology, UT Health Science Center, San Antonio, TX, USA

${ }^{*}$ Corresponding author:

Andrzej T. Slominski, MD, PhD, Department of Dermatology, University of Alabama at Birmingham, Birmingham, AL 35294. USA; e-mail: aslominski@uabmc.edu; phone: 205.934.5245 


\section{Supplementary Materials}

A
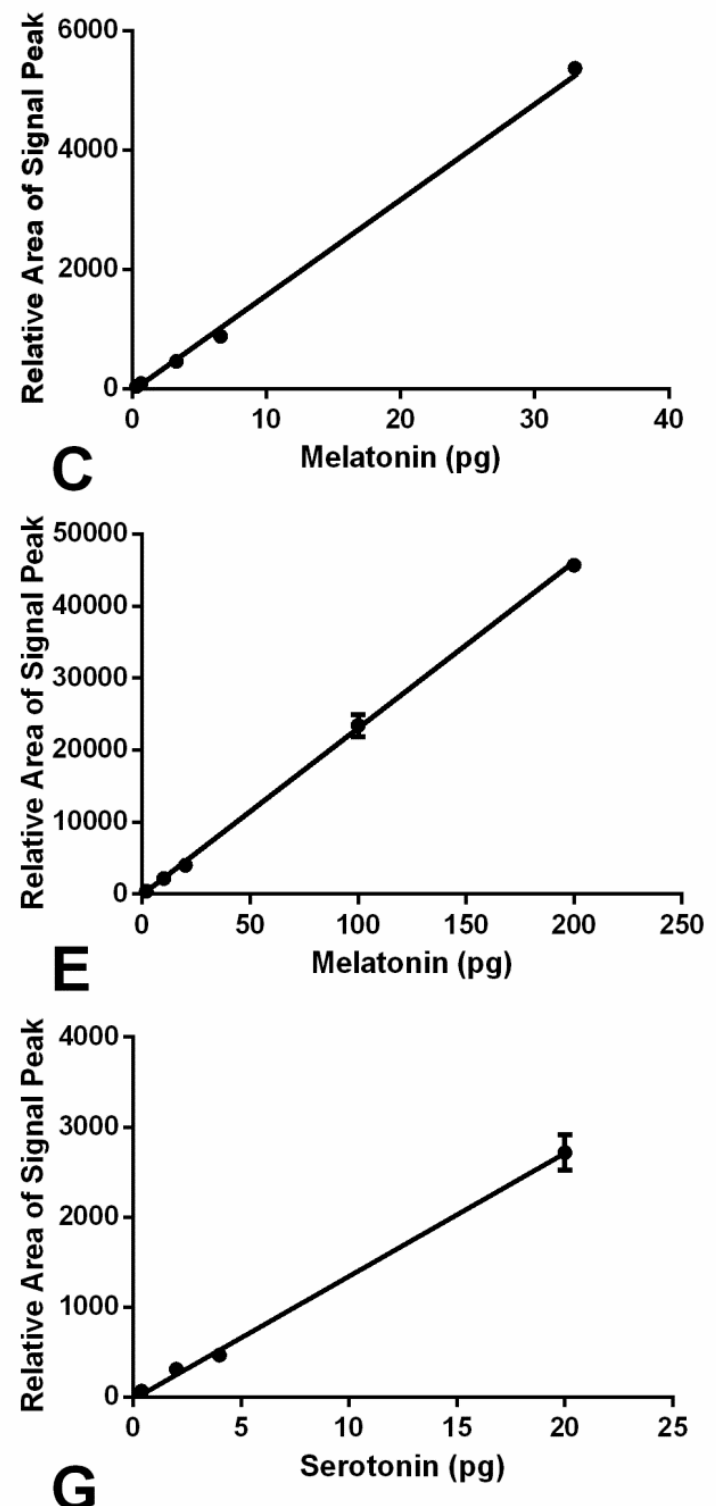

G

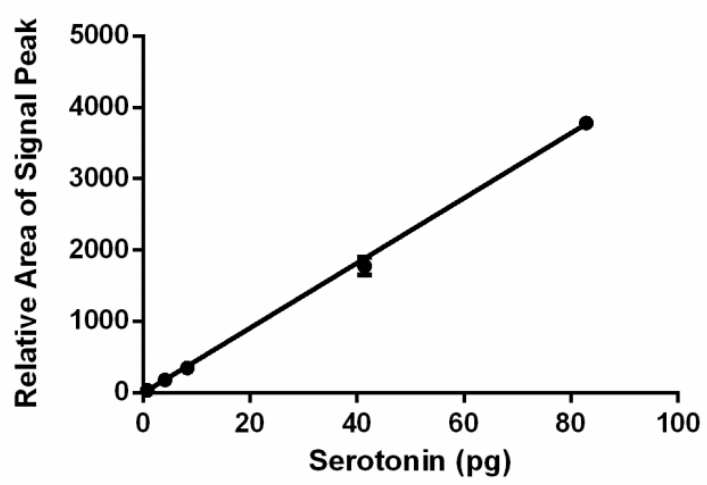

B
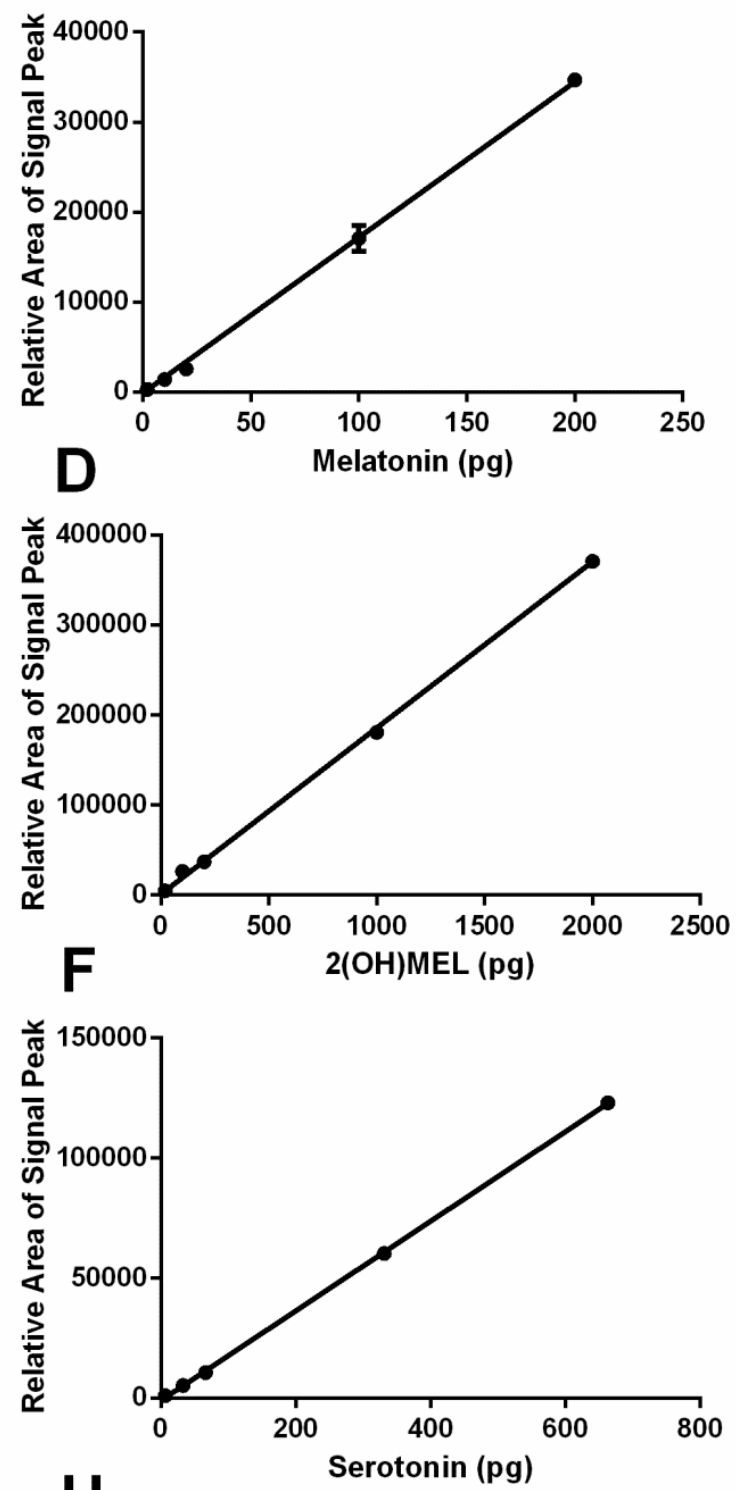

$\mathrm{H}$

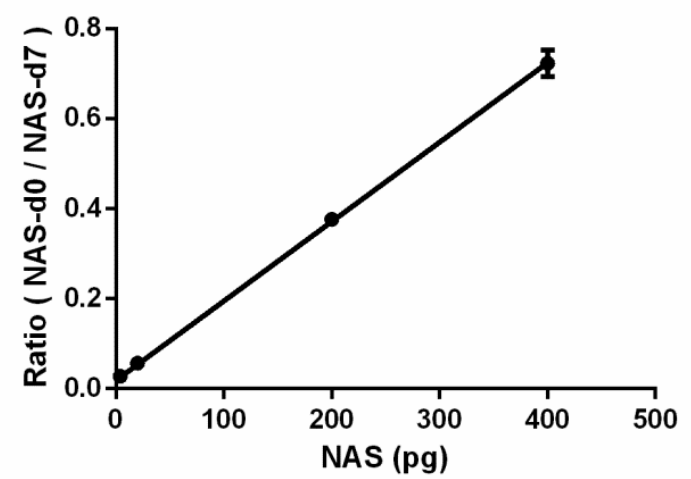


Figure S1. Standard curves for melatonin and its derivatives used for quantification. The peak area was calculated using Waters MassLynx ${ }^{\mathrm{TM}}$ Software with EIC of A, Melatonin, $m / z=174.1\left[\mathrm{M}+\mathrm{H}-\mathrm{NH}_{2} \mathrm{CH}_{3} \mathrm{CO}\right]^{+}$;

B, Melatonin, $m / z=233.1[\mathrm{M}+\mathrm{H}]^{+} ; \mathrm{C}$, Melatonin, $m / z=255.1[\mathrm{M}+\mathrm{Na}]^{+} ; \mathrm{D}, 2(\mathrm{OH}) \mathrm{MEL}, \mathrm{m} / \mathrm{z}=249.1$

$[\mathrm{M}+\mathrm{H}]^{+} ; \mathrm{E}$, Serotonin for commercial honey sample 1, $\mathrm{m} / \mathrm{z}=160.1\left[\mathrm{M}+\mathrm{H}-\mathrm{NH}_{3}\right]^{+} ; \mathrm{F}$, Serotonin for Australian honey and Polish commercial honey, $\mathrm{m} / \mathrm{z}=160.1\left[\mathrm{M}+\mathrm{H}-\mathrm{NH}_{3}\right]^{+}$; $\mathrm{G}$, Serotonin for commercial honey sample 2 and Polish natural honey, $m / z=160.1\left[\mathrm{M}+\mathrm{H}-\mathrm{NH}_{3}\right]^{+} ; \mathrm{H}, \mathrm{NAS}, \mathrm{m} / \mathrm{z}=160.1\left[\mathrm{M}+\mathrm{H}-\mathrm{NH}_{2} \mathrm{CH}_{3} \mathrm{CO}\right]^{+}$ and NAS-d7, $m / z=164.1\left[\mathrm{M}+\mathrm{H}-\mathrm{NH}_{2} \mathrm{CH}_{3} \mathrm{CO}\right]^{+}$
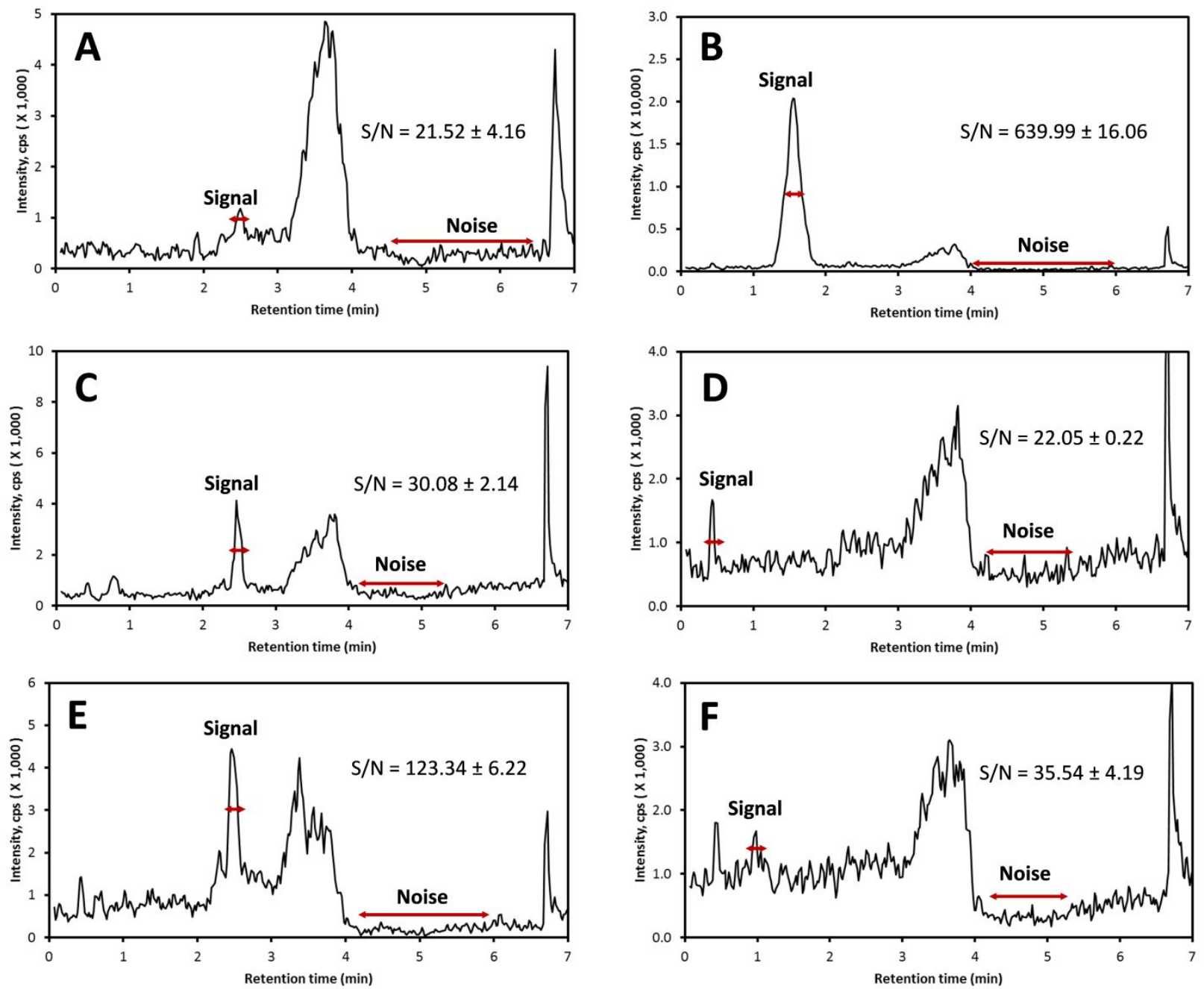

Figure S2. The limits of quantification (LOQ) were determined by calculating the ratio of signal/noise (S/N) using Waters MassLynx ${ }^{\mathrm{TM}}$ Software with EIC of A, 0.33 pg Melatonin, $\mathrm{m} / \mathrm{z}=174.1\left[\mathrm{M}+\mathrm{H}-\mathrm{NH}_{2} \mathrm{CH}_{3} \mathrm{CO}\right]^{+} ; \mathrm{B}$, 
$20 \mathrm{pg} 2(\mathrm{OH}) \mathrm{MEL}, \mathrm{m} / \mathrm{z}=249.1[\mathrm{M}+\mathrm{H}]^{+} ; \mathrm{C}, 2 \mathrm{pg}$ Melatonin, $\mathrm{m} / \mathrm{z}=233.1[\mathrm{M}+\mathrm{H}]^{+} ; \mathrm{D}, 0.4 \mathrm{pg}$ Serotonin, $\mathrm{m} / \mathrm{z}=$ $160.1\left[\mathrm{M}+\mathrm{H}-\mathrm{NH}_{3}\right]^{+} ; \mathrm{E}, 2 \mathrm{pg}$ Melatonin, $\mathrm{m} / \mathrm{z}=255.1[\mathrm{M}+\mathrm{Na}]^{+} ; \mathrm{F}, 0.4 \mathrm{pg} \mathrm{NAS}, \mathrm{m} / \mathrm{z}=160.1[\mathrm{M}+\mathrm{H}-$ $\left.\mathrm{NH}_{2} \mathrm{CH}_{3} \mathrm{CO}\right]^{+}$.
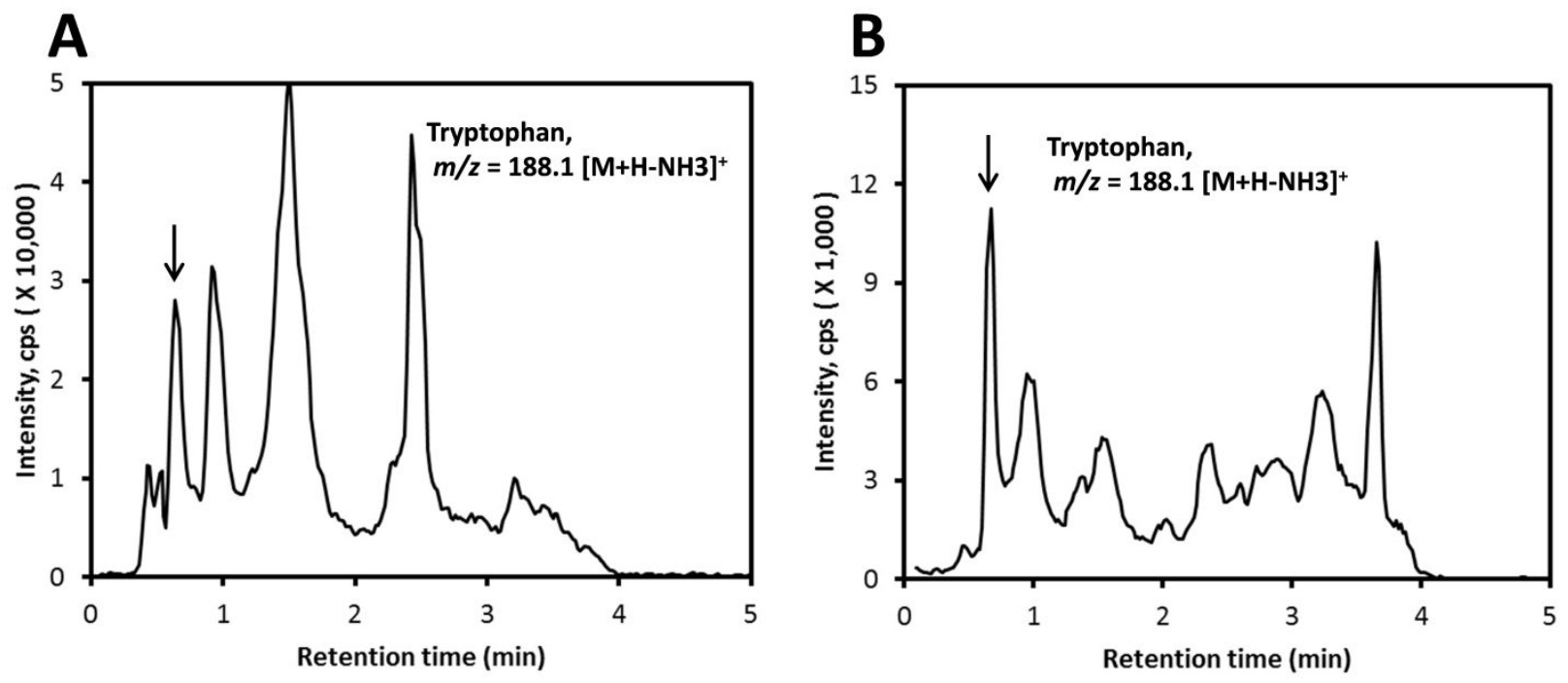

Figure S3. Detection of tryptophan in honey. A, Tryptophan in Australian honey; B, Tryptophan in commercial honey sample 1. 
Table S1. Recoveries of standard melatonin by methylene chloride and ethyl acetate extraction procedurs. Melatonin was added to water:honey mixture and samples were extracted with methylene chloride or ethyl acetate as described in the Methods, then analyzed by LC-MS.

\begin{tabular}{|l|c|c|c|}
\hline \multirow{2}{*}{ Samples } & \multicolumn{3}{|c|}{ Recovery (\%) } \\
\cline { 2 - 4 } & $m / z=233.1[\mathrm{M}+\mathrm{H}]^{+}$ & $m / z=255.1[\mathrm{M}+\mathrm{Na}]^{+}$ & $\begin{array}{l}\mathrm{m} / \mathrm{z}=174.1[\mathrm{M}+\mathrm{H}- \\
\left.\mathrm{NH}_{2} \mathrm{CH}_{3} \mathrm{CO}\right]^{+}\end{array}$ \\
\hline Melatonin Standard & 100 & 100 & $87.10 \pm 6.56$ \\
\hline $\begin{array}{l}\text { Methylene chloride } \\
\text { extraction }\end{array}$ & $77.17 \pm 9.24$ & $74.71 \pm 0.68$ & $66.26 \pm 4.07$ \\
\hline $\begin{array}{l}\text { Ethyl acetate } \\
\text { extraction }\end{array}$ & $58.38 \pm 4.21$ & $62.34 \pm 0.74$ & 800 \\
\hline
\end{tabular}

Table S2. Recoveries of standard serotonin by methylene chloride and ethyl acetate extraction procedures. Serotonin was added to water:honey mixture and samples were extracted with methylene chloride or ethyl acetate as describes in the Methods, then analyzed by LC-MS.

\begin{tabular}{|l|c|}
\hline Samples & $\begin{array}{c}\text { Recovery }(\%) \\
m / z=160.1\left[\mathrm{M}+\mathrm{H}-\mathrm{NH}_{3}\right]^{+}\end{array}$ \\
\hline Serotonin Standard & 100 \\
\hline Methylene chloride extraction & $15.20 \pm 0.31$ \\
\hline Ethyl acetate extraction & $133.69 \pm 3.72$ \\
\hline
\end{tabular}

Table S3. Recoveries of standard NAS by methylene chloride and ethyl acetate extraction procedures. Serotonin was added to water:honey mixture and samples were extracted with methylene chloride or ethyl acetate as describes in the Methods, then analyzed by LC-MS.

\begin{tabular}{|l|c|}
\hline Samples & $\begin{array}{c}\text { Recovery }(\%) \\
\end{array}$ \\
\hline NAS Standard & $\mathrm{m} / \mathrm{z}=160.1\left[\mathrm{M}+\mathrm{H}-\mathrm{NH}_{2} \mathrm{CH}_{3} \mathrm{CO}\right]^{+}$ \\
\hline Methylene Chloride extraction & 100 \\
\hline Ethyl Acetate extraction & $17.95 \pm 0.49$ \\
\hline
\end{tabular}

